\title{
Neuroprotective effects of the aerial parts of Polygala tenuifolia Willd extract on scopolamine-induced learning and memory impairments in mice
}

\author{
XIAO WANG ${ }^{*}$, DIAN ZHANG ${ }^{*}$, WEIHAO SONG, CHENG FEI CAI, ZIHAO ZHOU, \\ QI FU, XINGRONG YAN, YANJUN CAO and MINFENG FANG
}

Key Laboratory of Resource Biology and Biotechnology in Western China, Ministry of Education, Northwest University, Xi'an, Shaanxi 710069, P.R. China

Received October 31, 2019; Accepted July 10, 2020

DOI: $10.3892 /$ br.2020.1344

\begin{abstract}
Alzheimer's disease is a common neurodegenerative disease characterized by progressive cognitive dysfunction and behavioral impairment. Aerial parts of Polygala tenuifolia Willd (APT) is a traditional Chinese medicine used for the treatment of amnesia. The present study aimed to investigate the protective effects of APT on scopolamine-induced learning and memory impairments in mice. Scopolamine-induced mice were used to determine the effects of APT on learning and memory impairment. Mice were orally administered with APT $(25,50$ and $100 \mathrm{mg} / \mathrm{kg})$ and piracetam $(750 \mathrm{mg} / \mathrm{kg})$ for 14 days, and intraperitoneally injected with scopolamine $(2 \mathrm{mg} / \mathrm{kg}$ ) from days 8 to 14 . Morris water maze and step-down tests were performed to evaluate learning and memory. Levels of acetylcholine (ACh), choline acetyltransferase (ChAT), acetylcholinesterase (AChE), interleukin (IL)-1 $\beta$, IL-10 and brain-derived neurotrophic factor (BDNF) in the hippocampus and frontal cortex were measured by ELISA. Superoxide dismutase (SOD), malondialdehyde (MDA) and glutathione (GSH) were measured via biochemical detection. The results demonstrated that APT ameliorated learning and memory impairment in scopolamine-induced mice. Correspondingly, APT significantly increased ACh and ChAT levels in the hippocampus and prefrontal cortex of scopolamine-induced mice. Additionally, treatment with APT significantly increased BDNF and IL-10 levels, and decreased IL-1 $\beta$ and AChE levels
\end{abstract}

Correspondence to: Professor Minfeng Fang, Key Laboratory of Resource Biology and Biotechnology in Western China, Ministry of Education, Northwest University, 229 North Taibai Road, Xi'an, Shaanxi 710069, P.R. China

E-mail: fff885@126.com

*Contributed equally

Key words: aerial parts of Polygala tenuifolia Willd, cholinergic activity, brain-derived neurotrophic factor, neuroinflammation, oxidative stress in the same mice. Furthermore, APT significantly increased SOD activity and GSH content, and decreased MDA levels in brain tissue. These results indicated that APT may ameliorate learning and memory impairment by regulating cholinergic activity, promoting BDNF and inhibiting neuroinflammation and oxidative stress.

\section{Introduction}

Alzheimer's disease (AD), one of the most common forms of dementia, is caused by neuronal damage in the hippocampus and other brain regions that results in a decline in learning and memory $(1,2)$. For millions of individuals worldwide, AD treatment represents a large economic burden (3). Additionally, the number of cases is estimated to increase dramatically due to increasing lifespans and the lifestyle changes $(3,4)$.

A previous study determined that AD severity is associated with cholinergic neuron loss and markedly decreased levels of acetylcholine (ACh) in the brain (5). The cholinergic system serves an important role in the central and peripheral control of multiple cognitive processes (6). ACh is synthesized by choline acetyltransferase (ChAT) in cholinergic neurons and is hydrolyzed by acetylcholinesterase (AChE) (6). The decreased release of ACh following cholinergic neuron loss results in learning deficits. When $\mathrm{AD}$ occurs, cholinergic neurons in the hippocampus and prefrontal cortex are lost, resulting in the decreased synthesis, storage and release of Ach (7). This leads to a variety of clinical manifestations, primarily being memory and cognitive impairment (7). In the brains of patients with $\mathrm{AD}, \mathrm{ChAT}$ activity, a rate-limiting enzyme for ACh production in the cholinergic system of the frontal cortex and hippocampus, decreases significantly (8). Additionally, AD is associated with oxidative stress, inflammation and hyperhomocysteinemia (8).

It was reported that microglia and astrocytes release large numbers of pro-inflammatory cytokines, anti-inflammatory cytokines, chemokines and other related factors, including interleukin (IL)-1 $\beta$, IL-10 and monocyte chemoattractant protein-1 (9). IL-1 $\beta$ is a key regulator of neuroinflammation and is considered to be a potential genetic risk factor of dementia (10). It was observed that increased levels of IL-1 $\beta$ 
occur in the serum and cerebrospinal fluid of patients with AD and dementia (10). Elevated IL-1 $\beta$ also activates the microglia and astrocytes to further increase levels of cytokines and free radicals, leading to neurotoxicity (10). In patients with AD, levels of various oxidation products, including sugar, protein, lipid, nucleic acid and other macromolecule substances, are significantly increased in the cerebrospinal fluid and blood (11). Furthermore, markers of oxidative stress are increased in the urine of patients with AD (11). Although the brain accounts for $2 \%$ of total body mass, oxygen consumption accounts for $25 \%$ (11). Therefore, the brain is more sensitive to oxidative stress compared with other organs (8). A previous study revealed that oxidative stress serves an important role in the pathogenesis of AD (12). Excessive oxidative stress can result in lipid peroxidation on the membrane of nerve cells or organelles, and the destruction of protein nitration and nucleic acid, affecting the synaptic ability of nerve cells and leading to neuronal apoptosis (11).

The aerial parts of Polygala tenuifolia Willd (APT) is widely used in China as a traditional medicine for expectorant, sedative, anti-aging and anti-inflammatory properties (13). APT was first recorded in Divine Farmer's Classic of Materia Medica for the treatment of amnesia (13). The Clinical Manual of Traditional Chinese Medicine indicated that the medicinal sections of APT are the stem and leaves, which are prescribed using the name 'Xiaocao' (13). APT contains various active ingredients, including flavonoids and phenolic glycosides, which were reported to serve anti-inflammatory and anti-oxidative effects $(14,15)$. However, whether APT improves learning and memory in AD animal models is yet to be elucidated. Therefore, the current study aimed to investigate the ameliorating effects of APT extract on scopolamine-induced learning and memory impairment in mice. Morris water maze and step-down passive avoidance tests were implanted to achieve aims and explore potential mechanisms.

\section{Materials and methods}

Animals. Adult male Kunming mice $(20 \pm 2$ g) 12 weeks old were purchased from the Experimental Animal Center of Xi'an Jiaotong University (Xi'an, China) and maintained in specific pathogen-free conditions with free access to standardized feed and water. Animals were additionally housed at $23 \pm 2^{\circ} \mathrm{C}$ with a relative humidity of $40-60 \%$ under a 12 -h light/dark cycle. Animal experiments were approved by the Ethics Committee of Northwest University (Xi'an, China) and all efforts were made to minimize animal suffering and the number of rats used for experimentation. Ten mice were used in each group and a total of 60 mice were used in the experiments. Animal health and behavior were monitored daily.

Chemicals and drugs. Scopolamine hydrobromide (Scop; cat. no. 1601211) was purchased from Suicheng Pharmaceutical Co., Ltd. ELISA kits for ChAT (cat. no. 20171211), Ach (cat. no. 20170926) and AChE (cat. no. 20171027), brain-derived neurotrophic factor (BDNF; cat. no. 20171106), IL-10 (cat. no. 20171113) and IL-1 $\beta$ (cat. no. 20171113) were purchased from Yuduo Biological Technology Co., Ltd. Malondialdehyde (MDA; cat. no. 20171201), superoxide dismutase (SOD; cat. no. 20171201), glutathione (GSH; cat. no. 20171201) kits were obtained from the Nanjing Jiancheng Bioengineering Institute. Bicinchoninic acid protein assay kits (BCA; cat. no. 12G28C46) were purchased from Wuhan Boster Biological Technology Co., Ltd. Piracetam (cat. no. 2160402) were obtained from Renfu Pharmaceutical Co., Ltd.

Extract preparation. APT were collected from Chengcheng County (Shaanxi, China) in June of 2017 and authenticated by Professor MF Fang of Northwest University (Xi'an, China). APT (500 g) was ultrasonically extracted in triplicate with $50 \%(\mathrm{v} / \mathrm{v})$ ethanol ten times. The filtrate was subsequently mixed and concentrated in a vacuum at $50-60^{\circ} \mathrm{C}$ using a rotary evaporator. The dried residue obtained a yield of $125.5 \mathrm{~g}$, which was suspended in saline and subjected to in vivo experiments. The normal dose of aerial parts of Polygala tenuifolia Willd for human adults is $3.00 \mathrm{~g} / \mathrm{day}$ (16). Equivalently, the calculated dose of aerial parts of Polygala tenuifolia Willd based on respective body surface areas for mice is $0.4 \mathrm{~g} / \mathrm{kg} /$ day. The extraction rate of Polygala tenuifolia Willd is $25 \%$, and so the dose of aerial parts extract of Polygala tenuifolia Willd for mice is $100 \mathrm{mg} / \mathrm{kg} / \mathrm{day}$. Therefore, $100 \mathrm{mg} / \mathrm{kg} /$ day was chosen as the high dose, $50 \mathrm{mg} / \mathrm{kg} /$ day as the middle dose, and $25 \mathrm{mg} / \mathrm{kg} /$ day as the low dose in the present study.

Experimental design.Piracetam, a derivative of $\gamma$-aminobutyric acid, is a new type of memory promoting drug which is used to treat memory impairment and moderate brain dysfunction (17) and is selected as positive drug in the present study. After 7 days of acclimatization, mice were randomly divided into the following six groups $(\mathrm{n}=10)$ : The Scop group [ $2 \mathrm{mg} / \mathrm{kg} / \mathrm{day}$; intraperitoneal (ip) Scop] (18); the Piracetam (Pir) group [2 $\mathrm{mg} / \mathrm{kg} /$ day ip Scop and $750 \mathrm{mg} / \mathrm{kg} /$ day rally administered (ig) Pir] (19); the APT-25, APT-50 and APT-100 groups, which were treated with Scop ( $2 \mathrm{mg} / \mathrm{kg} /$ day ip) and 25, 50 and $100 \mathrm{mg} / \mathrm{kg} / \mathrm{day}$ (ig) APT, respectively; and the normal control (NC) group, which was treated with $5 \mathrm{ml} / \mathrm{kg}$ saline. Apart from the NC group, mice were injected ip with $2 \mathrm{mg} / \mathrm{kg}$ Scop for $1 \mathrm{~h}$ following administration of APT. Behavioral testing started 30 min following ip injection of Scop. The therapeutic drug of APT or Pir was administered for 14 days and Scop was continuously administered ip from days 8 to 14 (Fig. 1).

After 14 days of APT administration, the spatial learning and memory of Scop-induced mice was assessed via Morris water maze and step-down tests. Following behavioral assessment, pentobarbital sodium (200 mg/kg body weight, ip) was used for euthanasia, which was approved by the Ethical Committee of Northwest University. When the animal has no breath and no heartbeat, the brains were removed for biochemical analysis. The hippocampus and prefrontal cortex were dissected and stored at $-80^{\circ} \mathrm{C}$ until protein and ELISA analysis. The timeline of the present study is presented in Fig. 1.

Morris water maze test. A Morris water maze test was performed $30 \mathrm{~min}$ following Scop injection to evaluate memory-related behaviors. A large circular tank (diameter, $150 \mathrm{~cm}$; height, $35 \mathrm{~cm}$ ) was filled with water to a depth of $20 \mathrm{~cm}$ and maintained at a temperature of $25 \pm 2^{\circ} \mathrm{C}$. Ink was added into the water to prevent mice from seeing the platform. The platform $(10 \times 10 \mathrm{~cm})$ was fixed and hidden $2 \mathrm{~cm}$ below 


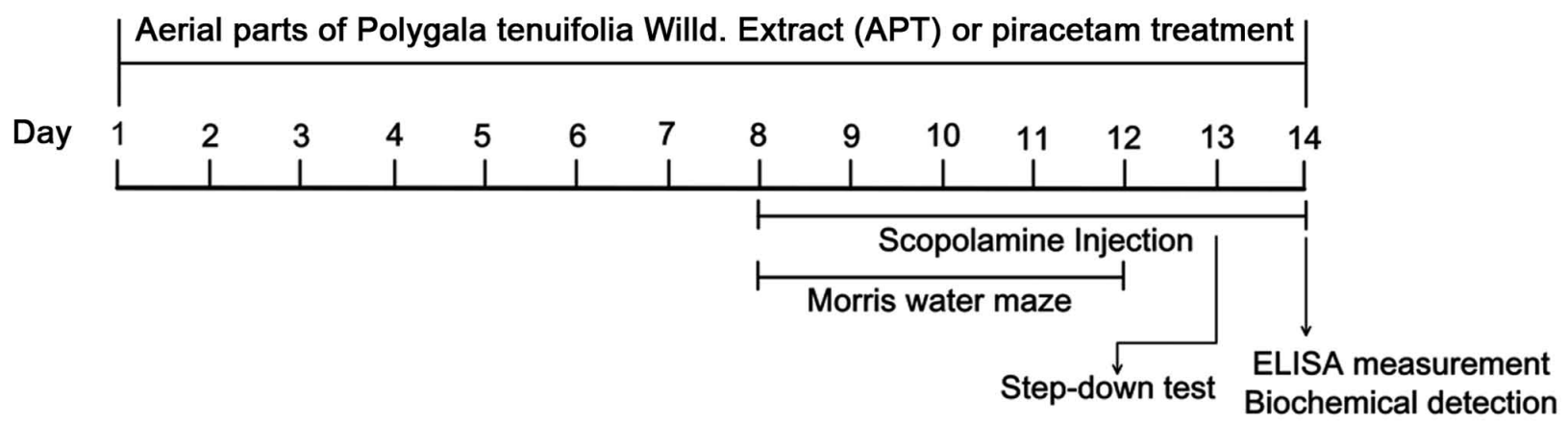

Figure 1. Timeline showing a summary of the experimental design. APT, aerial parts of Polygala tenuifolia Willd.

the water surface. During the acquisition-testing phase, mice completed four trials per day for four consecutive days during which they were left to locate the submerged platform. During these four consecutive days, starting positions were randomized for all mice. The mouse was permitted to rest on the platform for $10 \mathrm{sec}$, following which it was required to successfully locate the platform within $120 \mathrm{sec}$. The time between the first placement in water to platform location was recorded as the escape latency. If the mouse failed to find the platform within the permitted time, escape latency was recorded as $120 \mathrm{sec}$, after which the mouse was physically placed on the platform for $10 \mathrm{sec}$. On day 5 , the retention of spatial reference memory was recorded by a probe trial with the platform being removed from the pool. The number of platform crossings in the target quadrant was recorded as indices of spatial memory and the mice were allowed to swim freely for $120 \mathrm{sec}$.

Step-down test. After the Morris water maze test, the step-down test was performed. Six plastic boxes $\left(15 \times 15 \times 40 \mathrm{~cm}^{3}\right)$ with a high platform (diameter, $4.5 \mathrm{~cm}$; height, $4.5 \mathrm{~cm}$ ) were used for experimentation. Parallel caliber stainless steel bars were installed to produce a mild shock on the floor of the plastic box. All mice received training before the test and were allowed to acclimatize for the first $3 \mathrm{~min}$. Mice were placed on the platform and received continuous shocks (36 V) for $5 \mathrm{~min}$ as soon as they stepped down on the grid and floor. Mice would then step up to the platform. All animals underwent the same experimental procedure after $24 \mathrm{~h}$, during which the frequency of steps down to the grid floor and the time from the first step down to the platform were recorded. Results were obtained within $300 \mathrm{sec}$

Determination of ACh, AChE, ChAT, BDNF and IL-1 $\beta$ levels in the hippocampus and frontal cortex. Levels of ACh, AChE, ChAT, BDNF, IL-10 and IL-1 $\beta$ were measured using ELISA. All ELISA experimental procedures were performed in accordance with the manufacturer's protocol (Yuduo Biotech). Absorbance was measured using a microtiter plate reader (PerkinElmer, Inc.) at a wavelength of $450 \mathrm{~nm}$. Levels of indicators in the hippocampus and cortex were normalized to protein per $\mathrm{mg}$, and expressed as per $\mathrm{mg}$ protein.

Determination of MDA and GSH levels and SOD activity in murine brains. Brain tissue was homogenized in ice-cold $0.9 \%$ saline solution and centrifuged at $600 \mathrm{x}$ g for $10 \mathrm{~min}$ at $4^{\circ} \mathrm{C}$. The resulting supernatant was used to determine SOD, MDA and GSH levels using biochemical kits (Nanjing Jiancheng Bioengineering Institute) according to the manufacturer's protocol. The results of MDA, GSH and SOD activity were normalized to protein content.

Statistical analysis. All results were expressed as the mean $\pm \mathrm{SD}$ and data were analyzed using SPSS 24.0 (IBM Corp.). Datasets with multiple comparisons were evaluated using one-way ANOVA followed by Tukey's post hoc test. $\mathrm{P}<0.05$ was considered to indicate a statistically significant difference.

\section{Results}

Morris water maze test. As shown in Fig. 2A, the escape latencies of all groups gradually decreased over the course of acquisition testing, indicating that all animals were able to locate the platform. Compared with the NC group, the Scop group demonstrated a significant increase in escape latency over the four training days $(\mathrm{P}<0.01)$. On the second day of training, when compared with the Scop group $(105.08 \pm 17.58 \mathrm{sec})$, the Pir group $(81.12 \pm 19.99 \mathrm{sec})$ exhibited a significantly decreased escape latency $(\mathrm{P}<0.01)$. When compared with the same group, the APT-100 group $(80.80 \pm 11.08 \mathrm{sec})$ exhibited a significantly decreased escape latency $(\mathrm{P}<0.01)$. On the third day of training, when compared with the Scop group $(97.89 \pm 12.80 \mathrm{sec})$, the escape latencies of mice in the APT group were significantly decreased by $17.21 \%$ in the $50 \mathrm{mg} / \mathrm{kg}$ group $(\mathrm{P}<0.05)$ and $22.88 \%$ in the $100 \mathrm{mg} / \mathrm{kg}$ group $(\mathrm{P}<0.01)$. On day 4 , escape latencies in the 25,50 and $100 \mathrm{mg} / \mathrm{kg}$ APT groups were significantly decreased by $16.80(\mathrm{P}<0.05), 26.71(\mathrm{P}<0.01)$ and $30.57 \%(\mathrm{P}<0.01)$, respectively when compared with the Scop group $(87.63 \pm 8.49 \mathrm{sec})$.

In the probe trial, the number of crossing target quadrants was used to evaluate the retention of spatial memory. As shown in Fig. 2B, compared with the NC group, the Scop group exhibited a $58.36 \%$ decrease in the number of crossing target quadrants $(\mathrm{P}<0.01)$. Furthermore, the 25,50 and $100 \mathrm{mg} / \mathrm{kg}$ APT groups demonstrated a $48.36 \%(\mathrm{P}<0.05), 84.43 \%(\mathrm{P}<0.01)$ and $116.39 \%(\mathrm{P}<0.01)$ increase in the number of crossing target quadrants when compared with the Scop group. Pir groups demonstrated a significant increase in the number of crossing target quadrants when compared with the Scop group $(\mathrm{P}<0.01)$.

Step-down passive avoidance test. In the step-down test (Fig. 3), the Scop group demonstrated a 53.23\% decrease in 
A

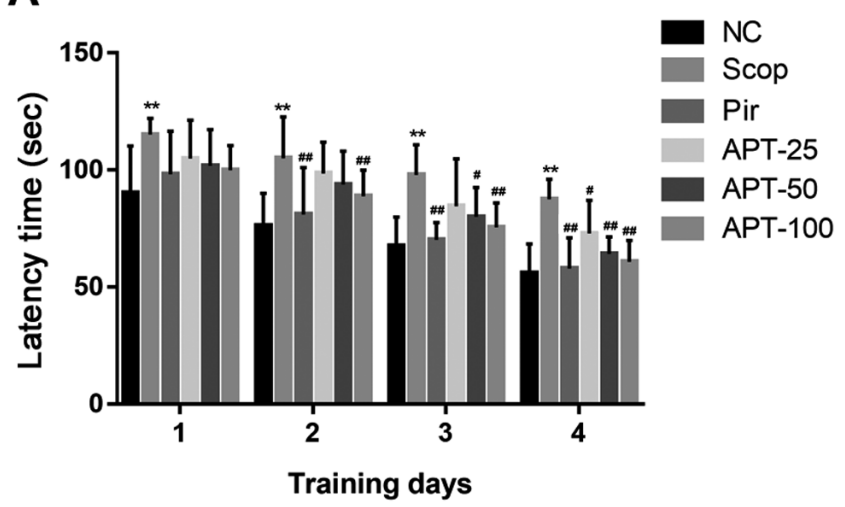

B

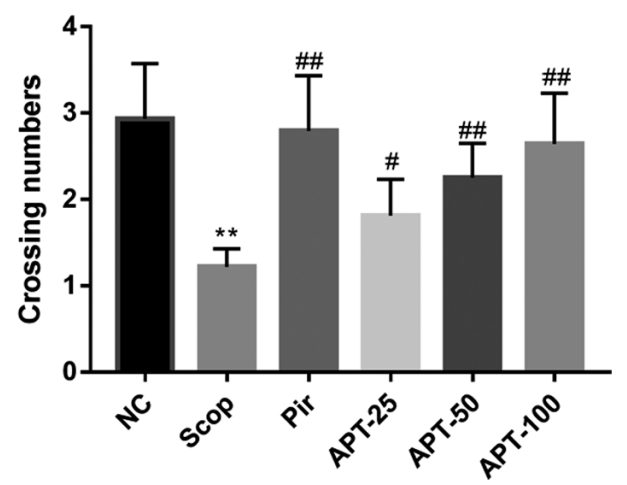

Figure 2. Effects of APT on spatial learning and memory in the Morris water maze test. (A) The escape latency in the Morris water maze test. (B) The number crossed on the former platform location. Data are presented as the mean \pm standard deviation. ${ }^{* *} \mathrm{P}<0.01$ vs. NC group. ${ }^{\#} \mathrm{P}<0.05$ and ${ }^{\# \#} \mathrm{P}<0.01$ vs. Scop. APT, aerial parts of Polygala tenuifolia Willd; Scop, scopolamine; Pir, piracetam; APT-25, 25 mg/kg APT, APT-50, 50 mg/kg APT; APT-100, 100 mg/kg APT; $\mathrm{NC}$, normal control.

A

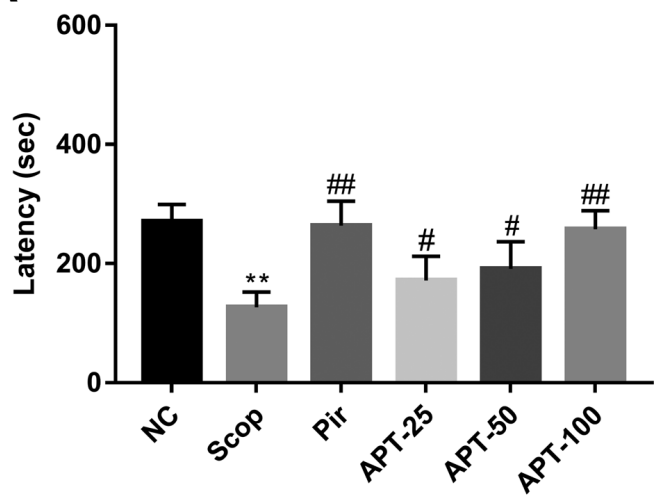

B

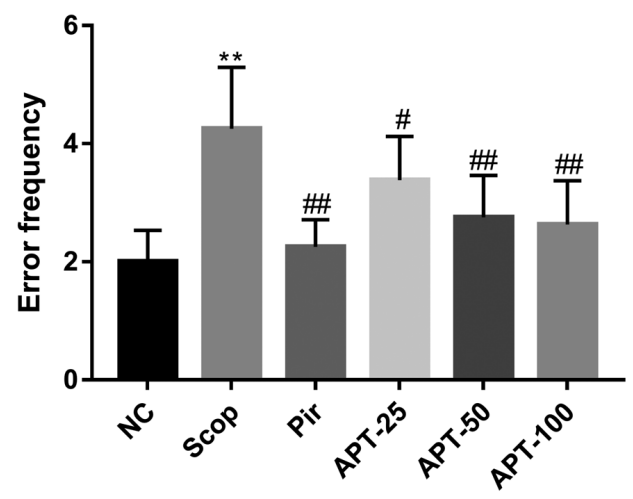

Figure 3. Effects of APT on the step-down passive avoidance test. (A) Step-down (A) latency (B) error frequency. Data are presented as the mean \pm standard deviation. ${ }^{* *} \mathrm{P}<0.01$ vs. NC group. ${ }^{\#} \mathrm{P}<0.05$ and ${ }^{\#} \mathrm{P}<0.01$ vs. Scop. APT, aerial parts of Polygala tenuifolia Willd; Scop, scopolamine; Pir, piracetam; APT-25, $25 \mathrm{mg} / \mathrm{kg}$ APT, APT-50, $50 \mathrm{mg} / \mathrm{kg}$ APT; APT-100, $100 \mathrm{mg} / \mathrm{kg}$ APT; NC, normal control.

step-down latency $(\mathrm{P}<0.01)$ and a $112.50 \%$ increase in error frequency $(\mathrm{P}<0.01)$ when compared with the $\mathrm{NC}$ group. Treatment with $25 \mathrm{mg} / \mathrm{kg}(171.75 \pm 40.58 \mathrm{sec} ; \mathrm{P}<0.05), 50 \mathrm{mg} / \mathrm{kg}$ $(191.13 \pm 45.38 \mathrm{sec} ; \mathrm{P}<0.05)$ and $100 \mathrm{mg} / \mathrm{kg}(257.88 \pm 30.77 \mathrm{sec}$; $\mathrm{P}<0.01)$ APT significantly increased the step-down latency and decreased the error frequency $(25 \mathrm{mg} / \mathrm{kg}, 3.38 \pm 0.74, \mathrm{P}<0.05$; $50 \mathrm{mg} / \mathrm{kg}, 2.75 \pm 0.71, \mathrm{P}<0.01 ; 100 \mathrm{mg} / \mathrm{kg}, 2.63 \pm 0.74, \mathrm{P}<0.01)$ when compared with the Scop group (latency, 126.63 \pm 25.75 sec; error frequency, $4.25 \pm 104)$. The Pir group, in comparison to the Scop group, also demonstrated significantly increased step-down latency $(263.63 \pm 41.12 \mathrm{sec} ; \mathrm{P}<0.01)$ and significantly decreased error frequency $(2.25 \pm 0.46 ; \mathrm{P}<0.01)$.

Effects of APT on ACh, AChE and ChAT levels in brain tissue. As shown in Fig. 4A and D, ACh levels in the hippocampus and frontal cortex were significantly lower in the Scop group compared with the NC group $(\mathrm{P}<0.01)$. Following APT administration, ACh levels were significantly increased in the hippocampus and frontal cortex compared with the Scop group $(\mathrm{P}<0.05$ and $\mathrm{P}<0.01)$. The Pir group also demonstrated a significant increase in ACh levels compared with the Scop group $(\mathrm{P}<0.01)$. There was no significant difference in $\mathrm{ACh}$,
AChE and ChAT content between the Pir and APT groups in the hippocampus and frontal cortex (Fig. 4).

As presented in Fig. 4B and E, the Scop group exhibited significantly higher levels of AChE compared with the NC group $(\mathrm{P}<0.01)$. APT treatment significantly decreased AChE activity compared with Scop-treated animals $(\mathrm{P}<0.05$ and $\mathrm{P}<0.01)$. A significant reduction was also observed in the Pir group compared with the Scop group $(\mathrm{P}<0.01)$.

ChAT levels in the hippocampus and frontal cortex of Scop-treated animals were significantly reduced compared with the NC group $(\mathrm{P}<0.01)$. Additionally, following treatment with APT, ChAT levels in the same areas of the brain were significantly increased $(\mathrm{P}<0.05$ and $\mathrm{P}<0.01)$ compared with the Scop group (Fig. 4C).

Effects of APT on BDNF in brain tissue. As shown in Fig. 5A, BDNF levels in the hippocampus and frontal cortex of mice were significantly decreased in the Scop group $(\mathrm{P}<0.01)$ compared with the NC group. Furthermore, APT treatment significantly increased BDNF levels compared with the Scop group $(\mathrm{P}<0.05)$. A significant increase in BDNF levels was also observed in the Pir group compared with the Scop group $(\mathrm{P}<0.01)$. 
A

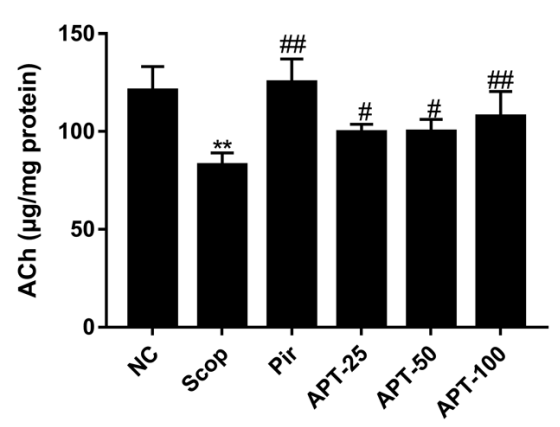

D

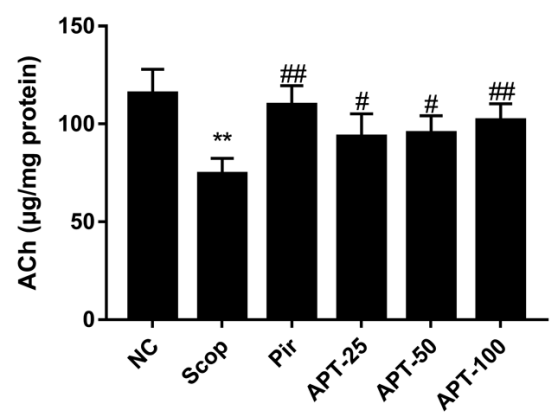

B

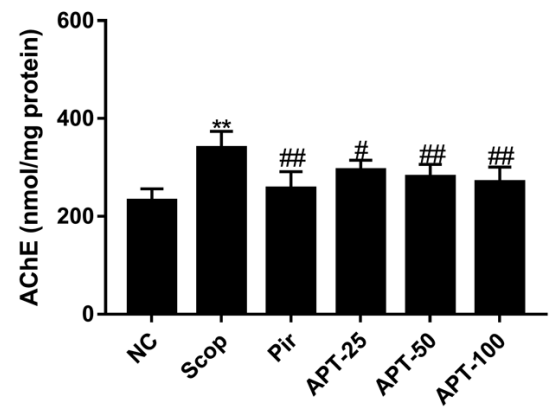

Cortex

E

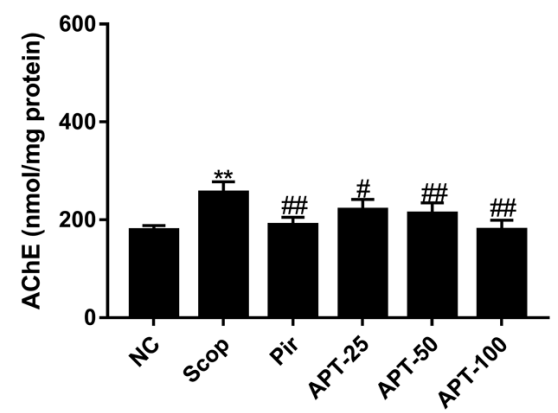

C

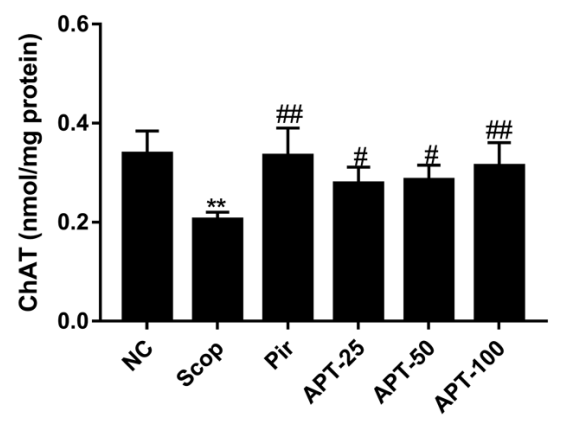

F

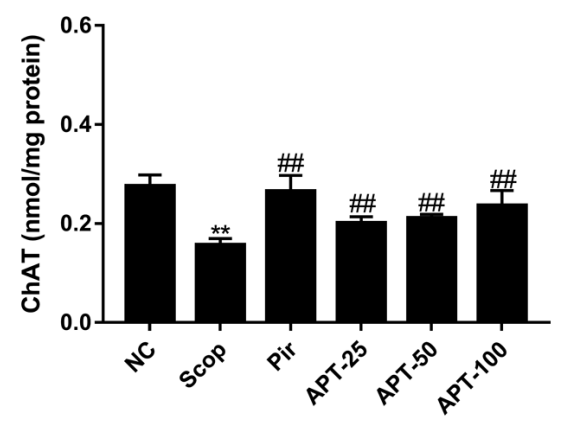

Figure 4. Effects of APT on Ach, AChE and ChAT levels in the hippocampus and prefrontal cortex in the Scop-induced model. (A) Ach, (B) AChE and (C) ChAT levels in the hippocampus. (D) Ach, (E) AChE, (F) and ChAT levels in the prefrontal cortex. Data are presented as the mean \pm standard deviation. ${ }^{* *} \mathrm{P}<0.01$ vs. NC group. ${ }^{~} \mathrm{P}<0.05$ and ${ }^{\# \#} \mathrm{P}<0.01$ vs. Scop. APT, aerial parts of Polygala tenuifolia Willd; Scop, scopolamine; Pir, piracetam; APT-25, $25 \mathrm{mg} / \mathrm{kg}$ APT, APT-50, $50 \mathrm{mg} / \mathrm{kg}$ APT; APT-100, $100 \mathrm{mg} / \mathrm{kg}$ APT; Ach, acetylcholine; AChE, acetylcholinesterase; ChAT, choline acetyltransferase; NC, normal control.

A

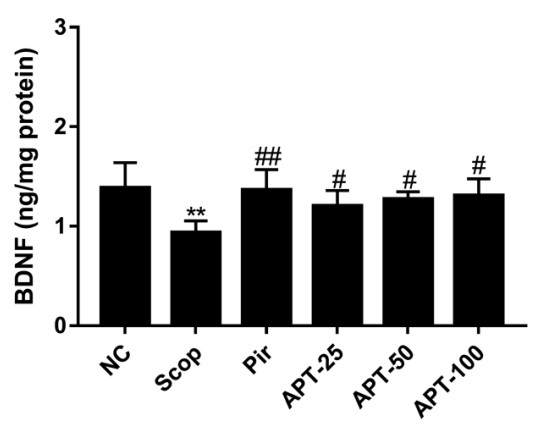

D

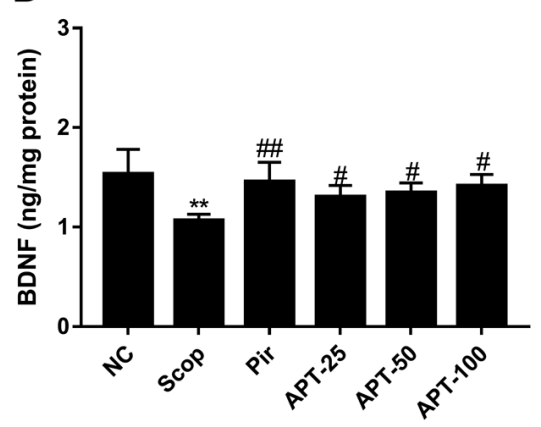

B

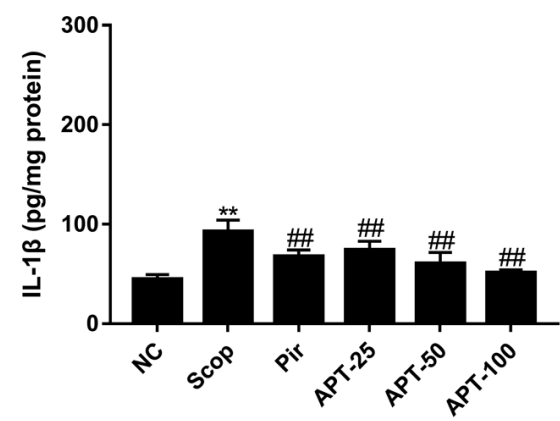

Cortex

E

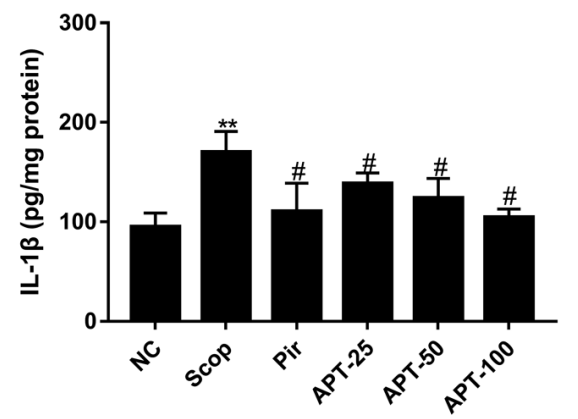

C

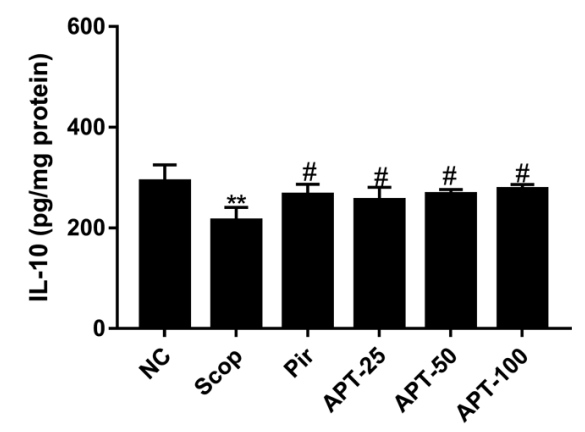

$\mathbf{F}$

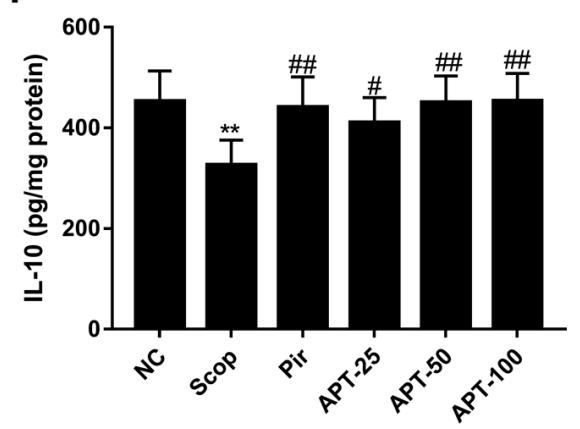

Figure 5. Effects of the aerial parts of APT on BDNF, IL-1 $\beta$ and IL-10 levels in the hippocampus and prefrontal cortex in the Scop-induced model. (A) BDNF, (B) IL-1 $\beta$ and (C) IL-10 levels in the hippocampus. (D) BDNF, (E) IL-1 $\beta$ and (F) IL-10 levels in the prefrontal cortex. Data are presented as the mean \pm standard deviation. ${ }^{* *} \mathrm{P}<0.01$ vs. NC group. ${ }^{\#} \mathrm{P}<0.05$ and ${ }^{\# \#} \mathrm{P}<0.01$ vs. Scop. APT, aerial parts of Polygala tenuifolia Willd; Scop, scopolamine; Pir, piracetam; APT-25, $25 \mathrm{mg} / \mathrm{kg}$ APT, APT-50, $50 \mathrm{mg} / \mathrm{kg}$ APT; APT-100, $100 \mathrm{mg} / \mathrm{kg}$ APT; NC, normal control; BDNF, brain-derived neurotrophic factor; IL, interleukin. 
A

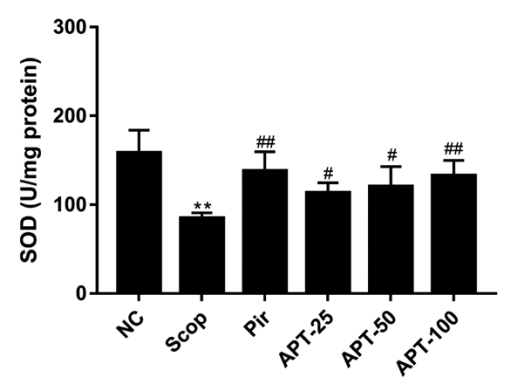

B

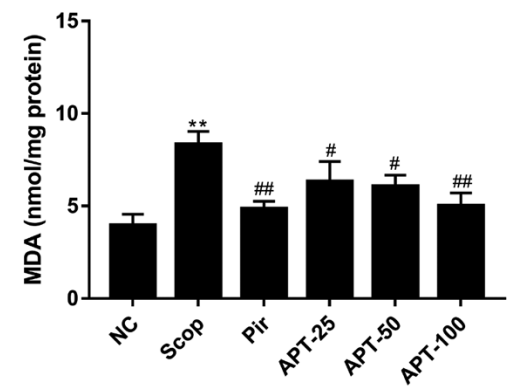

C

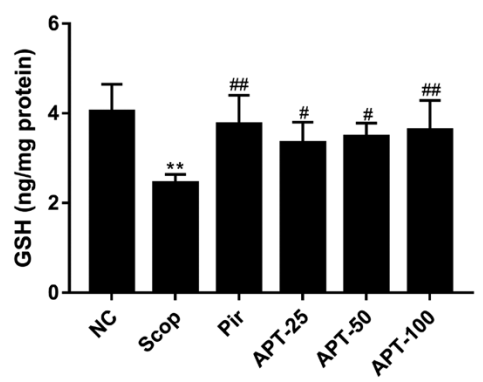

Figure 6. Effects of the aerial parts of APT on SOD, MDA and GSH levels in the Scop-induced model. (A) SOD, (B) MDA and (C) GSH levels. Data are presented as the mean \pm standard deviation. $n=10 .{ }^{* *} \mathrm{P}<0.01$ vs. NC group. ${ }^{\#} \mathrm{P}<0.05$ and ${ }^{\# \#} \mathrm{P}<0.01$ vs. Scop. APT, Polygala tenuifolia Willd extract; Scop, scopolamine; Pir, piracetam; APT-25, $25 \mathrm{mg} / \mathrm{kg}$ APT, APT-50, $50 \mathrm{mg} / \mathrm{kg}$ APT; APT-100, $100 \mathrm{mg} / \mathrm{kg}$ APT; NC, normal control; SOD, superoxide dismutase; MDA, malondialdehyde.

Effects of APT on IL-1 $\beta$ and IL-10 in brain tissue. IL-1 $\beta$ levels were significantly increased in the Scop group compared with the $\mathrm{NC}$ group (Fig. 5B; $\mathrm{P}<0.01$ ). Additionally, treatment with APT significantly decreased IL-1 $\beta$ activity compared with the Scop group $(\mathrm{P}<0.01)$.

IL-10 levels in the hippocampus and frontal cortex of mice were significantly lower in the Scop group compared with the NC group (Fig. 5C and F; P<0.01). However, treatment with APT significantly increased IL-10 levels when compared with the Scop group $(\mathrm{P}<0.05$ and $\mathrm{P}<0.01)$.

Effects of APT on SOD activity and MDA and GSH levels in brain tissue. As shown in Fig. 6A, SOD activity in the Scop group significantly decreased in the brain tissue compared with the NC group $(\mathrm{P}<0.01)$. Treatment with $25 \mathrm{mg} / \mathrm{kg}$ (33.89\%; $\mathrm{P}<0.05), 50 \mathrm{mg} / \mathrm{kg}(42.16 \% ; \mathrm{P}<0.05)$ and $100 \mathrm{mg} / \mathrm{kg}(56.78 \%$; $\mathrm{P}<0.01)$ APT significantly increased SOD activity compared with the Scop group. Furthermore, MDA levels in the remaining brain tissue were significantly increased in the Scop group compared with the NC group $(\mathrm{P}<0.01$; Fig. 6B). However, APT treatment demonstrated a significant dose-dependent decrease in MDA levels compared with the Scop group $(\mathrm{P}<0.05$ and $\mathrm{P}<0.01$; Fig. 6B). As presented in Fig. 6C, GSH levels significantly decreased in the Scop group compared with the NC group $(\mathrm{P}<0.01)$. However, following treatment with $25 \mathrm{mg} / \mathrm{kg}$ $(36.89 \% ; \mathrm{P}<0.05), 50 \mathrm{mg} / \mathrm{kg}(42.21 \% ; \mathrm{P}<0.05)$ and $100 \mathrm{mg} / \mathrm{kg}$ $(48.36 \%$; $\mathrm{P}<0.01)$ APT, GSH levels significantly increased compared with Scop-treated mice (Fig. 6C).

\section{Discussion}

The present study assessed the effects of APT treatment on learning and memory impairment in Scop-induced mice. The results revealed that Scop administration induced spatial learning and memory impairment, which was subsequently reversed following APT treatment. It was also demonstrated that APT treatment increased step-down latency and decreased error frequency in Scop-induced mice. Additionally, APT treatment significantly increased ACh and ChAT levels and decreased $\mathrm{AChE}$ content in the hippocampus and prefrontal cortex of Scop-induced mice. The results of the current study also demonstrated that APT significantly increased BDNF and IL-10 levels, and decreased IL-1 $\beta$ levels in the hippocampus and prefrontal cortex of Scop-induced mice. Furthermore, APT significantly increased SOD activity and decreased MDA and GSH levels. In the present study, piracetam was selected as the positive drug, which has been originally used as medication for AD and dementia (17). Its mechanism of action bases on facilitating activity in neurotransmitter systems such as cholinergic, dopaminergic, noradrenergic systems, and maintaining neuron receptors (19). In the present study, piracetam or APT can significantly ameliorate learning and memory impairment by regulating cholinergic activity, promoting BDNF and inhibiting neuro-inflammation and oxidative stress. The results indicated that APT may be used as a neuroprotective drug for Scop-induced learning and memory impairment.

The cholinergic system is considered to be the epicenter of diseases characterized by cognitive deficits (7). Nicotinic and muscarinic receptors, the cholinergic receptors of the brain, are involved in memory formation (17). Scopolamine, a muscarinic cholinergic receptor antagonist, impairs learning and memory in humans and rodents, particularly during learning acquisition and short-term memory (18). Furthermore, scopolamine significantly increased AChE and MDA levels in the cortex and hippocampus, increasing oxidative stress in the brain $(20,21)$. Scopolamine has been widely accepted as a pharmacological model to study cognitive impairment (22). Therefore, the present study utilized a murine scopolamine model to evaluate the ameliorating amnesia effects of APT. The results demonstrated that APT treatment not only reversed the scopolamine-induced increase in escape latency but also increased the number of crossings into the target quadrant. Furthermore, APT increased step-down latency and decreased error frequency of scopolamine-induced mice. The results indicated that APT improved scopolamine-induced learning and memory impairment.

Cholinergic neurons are primarily distributed in the basal nucleus, the diagonal band of Broca and the medial septal nucleus of the brain (17). These neurons transport large quantities of ACh to the cerebral cortex and hippocampus via projecting fibers (16). Previous studies have demonstrated that $\mathrm{ACh}$, which is synthesized by ChAT in cholinergic neurons and hydrolyzed by $\mathrm{AChE}$ after its release, is vital to learning and memory processes (20-22). In patients with $A D$, enzymes involved in the cholinergic system are particularly involved and the activity of ChAT, the rate-limiting enzyme for ACh 
production in frontal cortex and hippocampus, is significantly decreased (7). Thus, AChE inhibition may serve as a therapeutic target for the treatment of AD (5). In the current study, treatment with APT significantly inhibited AChE activity and effectively increased the levels of ChAT and ACh, and ameliorated learning and memory impairment.

BDNF is an important member of the neurotrophin family and serves an important role in the differentiation, proliferation, nutrition and maturation of neurons (23). BDNF can also enhance synaptic connections, affect neuronal plasticity and influence neurotransmitter synthesis $(23,24)$. The results of the present study indicated that treatment with APT significantly increased BDNF levels in the hippocampus and frontal cortex of mice. Previous studies have demonstrated that systemic inflammation is closely associated with dementia $(25,26)$. IL-1 $\beta$, a pro-inflammatory cytokine, is vital for the development of a complex hormonal and cellular inflammatory cascade (9). It was reported that IL-1 $\beta$ can promote the progression of neurodegenerative diseases by inducing nitric oxide production and declining cholinergic function, in turn improving AChE activity $(10,27)$. Furthermore, IL-10, an important anti-inflammatory cytokine, serves an important role in recovery from brain injury and may reduce the risk of $\mathrm{AD}(9,28)$. If cytokines in the brain are dysregulated, they may cause corresponding pathologic changes, including inflammatory effects and oxidative stress, affecting neural homeostasis $(27,29,30)$. In the present study, APT treatment significantly decreased IL- $1 \beta$ levels and increased IL-10 levels in the hippocampus and frontal cortex of scopolamine-treated mice. The results indicated that APT improved learning and memory via pro-inflammatory and anti-inflammatory mediators in scopolamine-induced mice.

Scopolamine not only interrupts cholinergic neurotransmitters, but also triggers oxidative stress in the brain, which are associated with the pathogenesis of AD (18). Oxidative stress can cause damage to brain cells and other neural tissues, leading to aging and untimely cell apoptosis (11). The imbalance between reactive oxygen species production and elimination by antioxidant enzymes results in lipid peroxidation, cellular signaling pathway and gene regulation alterations (31). MDA, produced via lipid peroxidation in free radicals, causes the cross-linking polymerization of protein, nucleic acid and other living macromolecules, and also results in cytotoxicity (12). It is one of the most important products of membrane lipid peroxidation and serves as an indicator of oxidative stress $(12,32)$. A previous study measured antioxidant enzyme activity and peroxide content in the brain tissue of patients with AD, the results of which revealed that GSH and SOD activities were significantly lower compared with healthy individuals, while MDA levels were significantly increased (33). The results further demonstrated that the production of free radicals in patients with $\mathrm{AD}$ was increased, inducing serious damage and decreasing the function of neurons (33). Lipid peroxide increases membrane fluidity on the surface of the cell membrane and causes neurodegenerative lesions (12). It was reported that APT contains various active ingredients such as flavonoids and phenolic glycosides, especially mangiferin, which is able to protect the central nervous system from oxidative stress, mitochondrial dysfunction and neuroinflammation and plays a role in improving declined memory and cognition (34). In the present study, scopolamine treatment significantly increased MDA levels and decreased SOD and GSH levels. However, following treatment with APT, SOD activity and GSH levels were significantly increased, and MDA levels were significantly decreased, indicating that APT improved learning and memory impairment through an anti-oxidant effect.

The administration of APT significantly decreased escape latency, increased the number of crossings into the target quadrant, increased step-down latency and decreased error frequency in scopolamine-induced mice. APT also facilitated central cholinergic activity by inhibiting AChE and increasing $\mathrm{ACh}$ and ChAT levels in the brains of mice. Treatment with APT also significantly increased BDNF and IL-10 levels, and decreased IL-1 $\beta$ levels in murine brains. Furthermore, APT significantly increased SOD activity and decreased levels of MDA and GSH. The results indicated that APT treatment may ameliorate learning and memory impairment by regulating cholinergic activity, promoting BDNF and inhibiting neuroinflammation and oxidative stress.

\section{Acknowledgements}

Not applicable.

\section{Funding}

This study was supported by the Key Research and Development Plan of Shaanxi Province (grant no. 2018ZDXM-SF-014), the Shaanxi Provincial Education Department Serves Local Special Projects (grant no. 2018JC032), the open funding of Key Laboratory of Resource Biology and Biotechnology in Western China, Ministry of Education, Northwest University (grant no. ZSK2018006), and the Public Health Specialty of the Department of Traditional Chinese Medicine (grant no. 2019-39).

\section{Authors' contributions}

MF designed the study and provided financial support. XW, WS, CFC, ZZ and QF performed the experiments and analyzed data. XW, DZ, MF and YC performed the experiments, wrote and edited the manuscript. XY provided technical support and performed the experiments. All authors read and approved the final manuscript.

\section{Availability of data and materials}

All data generated or analyzed during this study are included in this published article.

\section{Ethics approval and consent to participate}

All experiments were performed according to the protocol approved by the Ethics Committee of Northwest University (Xi'an, China).

\section{Patient consent for publication}

Not applicable. 


\section{Competing interests}

The authors declare that they have no competing interests.

\section{References}

1. Zvěřová M: Clinical aspects of Alzheimer's disease. Clin Biochem 72: 3-6, 2019.

2. Arranz AM and De Strooper B: The role of astroglia in Alzheimer's disease: Pathophysiology and clinical implications. Lancet Neurol 18: 406-414, 2019.

3. Cheng X, Wu J, Geng M and Xiong J: Role of synaptic activity in the regulation of amyloid beta levels in Alzheimer's disease. Neurobiol Aging 35: 1217-1232, 2014.

4. Ballard C, Gauthier S, Corbett A, Brayne C, Aarsland D and Jones E: Alzheimer's disease. Lancet 377: 1019-1031, 2011.

5. Teipel S, Heinsen H, Amaro EJ, Grinberg LT, Krause B and Grothe M; Alzheimer's disease neuroimaging initiative: cholinergic basal forebrain atrophy predicts amyloid burden in Alzheimer's disease. Neurobiol Aging 35: 482-491, 2014.

6. McKeever PM, Kim T, Hesketh AR, MacNair L, Miletic D, Favrin G, Oliver SG, Zhang Z, St George-Hyslop P and Robertson J: Cholinergic neuron gene expression differences captured by translational profiling in a mouse model of Alzheimer's disease. Neurobiol Aging 57: 104-119, 2017.

7. Klaassens BL, van Gerven JMA, Klaassen ES, van der Grond J and Rombouts S: Cholinergic and serotonergic modulation of resting state functional brain connectivity in Alzheimer's disease. NeuroImage 199: 143-152, 2019.

8. Ulep MG, Saraon SK and McLea S: Alzheimer disease. J Nurse Pract 14: 129-135, 2018

9. Ozben T and Ozben S: Neuro-inflammation and anti-inflammatory treatment options for Alzheimer's disease. Clin Biochem 72 87-89, 2019.

10. Morgan AR, Touchard S, Leckey C, O'Hagan C, Nevado-Holgado AJ; NIMA Consortium, Barkhof F, Bertram L, Blin O, Bos I, et al: Inflammatory biomarkers in Alzheimer's disease plasma. Alzheimers Dement 15: 776-787, 2019.

11. Jomova K, Vondrakova D, Lawson M and Valko M: Metals, oxidative stress and neurodegenerative disorders. Mol Cell Biochem 345: 91-104, 2010.

12. Pena-Bautista $C$, Baquero $M$, Vento $M$ and Chafer-Pericas $C$ : Free radicals in Alzheimer's disease: Lipid peroxidation biomarkers Clin Chim Acta 491: 85-90, 2019.

13. Lin Z, Gu J, Xiu J, Mi T, Dong J and Tiwari JK: Traditional chinese medicine for senile dementia. Evid Based Complement Alternat Med 2012: 692621, 2012.

14. Shi TX, Li Y and Jiang Y: Isolation of flavonoids from the aerial parts of Polygala tenuifolia Willd. and their antioxidant activities. J Chin Pharmaceut Sci 22: 36-39, 2013.

15. Yang XJ, Zou PP, Tu PF and Jiang Y: HPLC determination of mangiferin in the leaves of Aquilaria sinensis and the different aerial parts of Polygala tenuifolia. Chinese J Pharmaceut Anal 32: 1175-1178, 2012.

16. Medicine NAoTC: Chinese Materia Medica. Shanghai Scientific \& Technical Publishers, 1999.

17. Wilms W, Woźniak-Karczewska M, Corvini PF and Chrzanowski Ł: Nootropic drugs: Methylphenidate, modafinil and piracetam-population use trends, occurrence in the environment, ecotoxicity and removal methods-A review. Chemosphere 233: 771-785, 2019.
18. Pepeu G and Grazia Giovannini M: The fate of the brain cholinergic neurons in neurodegenerative diseases. Brain Res 1670: 173-184, 2017.

19. Krivinko JM, Koppel J, Savonenko A and Sweet RA: Animal models of psychosis in Alzheimer disease. Am J Geriatr Psychiatr 28: 1-19, 2020

20. Tao L, Xie J, Wang Y, Wang S, Wu S, Wang Q and Ding H: Protective effects of aloe-emodin on scopolamine-induced memory impairment in mice and $\mathrm{H}_{2} \mathrm{O}_{2}$-induced cytotoxicity in PC12 cells. Bioorg Med Chem Lett 24: 5385-5389, 2014.

21. Jeong EJ, Lee KY, Kim SH, Sung SH and Kim YC: Cognitive-enhancing and antioxidant activities of iridoid glycosides from Scrophularia buergeriana in scopolamine-treated mice. Eur J Pharmacol 588: 78-84, 2008.

22. Klinkenberg I and Blokland A: The validity of scopolamine as a pharmacological model for cognitive impairment: A review of animal behavioral studies. Neurosci Biobehav Rev 34: 1307-1350, 2010.

23. Hu W, Feng Z, Xu J, Jiang Z and Feng M: Brain-derived neurotrophic factor modified human umbilical cord mesenchymal stem cells-derived cholinergic-like neurons improve spatial learning and memory ability in Alzheimer's disease rats. Brain Res 1710: 61-73, 2019.

24. Bekinschtein P, Cammarota $M$ and Medina JH: BDNF and memory processing. Neuropharmacology 76: 677-683, 2014

25. Tanila H: The role of BDNF in Alzheimer's disease. Neurobiol Dis 97: 114-118, 2017.

26. Tapia-Arancibia L, Aliaga E, Silhol M and Arancibia S: New insights into brain BDNF function in normal aging and Alzheimer disease. Brain Res Rev 59: 201-220, 2008.

27. Kinney JW, Bemiller SM, Murtishaw AS, Leisgang AM, Salazar AM and Lamb BT: Inflammation as a central mechanism in Alzheimer's disease. Alzheimers Dement 4: 575-590, 2018

28. Subhramanyam CS, Wang $\mathrm{C}, \mathrm{Hu} \mathrm{Q}$ and Dheen ST: Microglia-mediated neuroinflammation in neurodegenerative diseases. Semin Cell Dev Biol 97: 112-120, 2019.

29. Hampel H, Vergallo A, Aguilar LF, Benda N, Broich K, Cuello AC, Cummings J, Dubois B, Federoff HJ, Fiandaca M, et al: Precision pharmacology for Alzheimer's disease. Pharmacol Res 130: 331-365, 2018.

30. Faria MC, Goncalves GS, Rocha NP, Moraes EN, Bicalho MA, Gualberto Cintra MT, Jardim de Paula J, José Ravic de Miranda LF, Clayton de Souza Ferreira A, Teixeira AL, et al: Increased plasma levels of BDNF and inflammatory markers in Alzheimer's disease. J Psychiatr Res 53: 166-172, 2014.

31. Schrag M, Mueller C, Zabel M, Crofton A, Kirsch WM, Ghribi O, Squitti R and Perry G: Oxidative stress in blood in Alzheimer's disease and mild cognitive impairment: A meta-analysis. Neurobiol Dis 59: 100-110, 2013.

32. Tramutola A, Lanzillotta C, Perluigi M and Butterfield DA: Oxidative stress, protein modification and Alzheimer disease. Brain Res Bull 133: 88-96, 2017.

33. Jiang T, Sun Q and Chen S: Oxidative stress: A major pathogenesis and potential therapeutic target of antioxidative agents in Parkinson's disease and Alzheimer's disease. Prog Neurobiol 147: $1-19,2016$

34. Feng ST, Wang ZZ, Yuan YH, Sun HM, Chen NH and Zhang Y: Mangiferin: A multipotent natural product preventing neurodegeneration in Alzheimer's and Parkinson's disease models. Pharmacol Res 146: 104336, 2019. 